14. Comel M. Ichtyosis linearis circumflexa. Dermatologica 1949; 98 : 133-6.

15. Netherton $E W$. A unique case of Trichorrexis Invaginata. Arch Dermatol $1958 ; 78: 483-7$.

16. Judge MR, Morgan G, Harper JL. A clinical and immunological study of Netherton's syndrome. $\mathrm{Br}$ J Dermatol 1994 ; 131: 615-21.

17. Chavanas S, C Bodemer, A Rochat, et al., Mutations in SPINK5, encoding a serine protease inhibitor, cause Netherton syndrome. Nat Genet $2000 ; 25: 141-2$.

18. Bitoun $\varepsilon$, Chavanas $S$, Irvine AD, et al. Netherton syndrome: disease expression and spectrum of SPINK5 mutations in 21 families. J Invest Dermatol 2002; $118: 352-61$.
19. Bitoun $\varepsilon$, Micheloni A, Lamant L, et al., LEKTI proteolytic processing in human primary keratinocytes, tissue distribution and defective expression in Netherton syndrome. Hum Mol Genet $2003 ; 12: 2417-30$.

20. Descargues P, Deraison C, Bonnart C, et al. Spink5deficient mice mimic Netherton syndrome through degradation of desmoglein 1 by epidermal protease hyperactivity. Nat Genet $2005 ; 37:$ 56-65.

21. Bonnart C, Deraison C, Lacroix M, et al. Elastase 2 is expressed in human and mouse epidermis and impairs skin barrier function in Netherton syndrome through filaggrin and lipid misprocessing. J Clin Invest 2010; $120: 871-82$

\title{
NOUVELLE
}

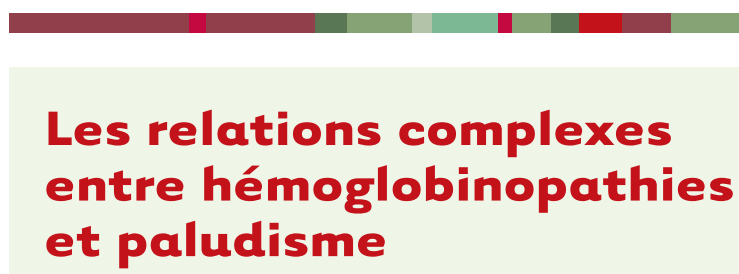

Dominique Labie

Protection contre le paludisme -

\section{Données historiques}

Les relations entre les hémoglobinopathies - l'hémoglobine $\mathrm{S}(\mathrm{HbS})$ en particulier, responsable de la drépanocytose - et le paludisme à Plasmodium falciparum ont été évoquées de longue date. Constatées par Beet dès 1949 [1] en Rhodésie (actuel Zimbabwe), elles ont permis de formuler l'hypothèse d'un polymorphisme équilibré (balanced polymorphism) basé sur la constatation, malgré le décès précoce de la majorité des drépanocytaires SS homozygotes, d'une survie préférentielle des hétérozygotes $A S$, qui acquièrent un niveau de protection atteignant $60-80 \%$ contre les complications sévères de la malaria [2]. Cet équilibre a pu être présenté comme l'exemple le plus typique de sélection naturelle [3]. La quasi-identité de la distribution géographique de l'HbS en Afrique et de celle des zones d'impaludation était un argument important en faveur de cette hypothèse. Par la suite, divers travaux ont cherché à déterminer les causes moléculaires et cellulaires de cette survie. La première explication remonte à 1970: Luzzatto constatait alors que la vitesse de falciformation des globules rouges (GR) in vitro était 2 à 3 fois plus rapide pour les globules rouges (GR) AS parasités, ce qui entrầnait la mort «suicidaire» du parasite et la séquestration des GR au niveau de la rate [4]. Le phénomène fut ensuite reproduit en montrant que le parasite survit moins bien dans des cultures de GR AS maintenus dans des conditions de p02 et de $\mathrm{pH}$ comparables à celles du sang veineux [5]. In vivo, dans la circulation veineuse profonde où la p02 est basse, les parasites sous forme de schizontes forment à la surface des GR des protubérances (knobs) qui adhèrent aux parois des microveinules, entraînant là encore l'élimination de la cellule parasitée. On a aussi suggéré le rôle d'une perte de potassium et de la déshydratation cellulaire qui s'ensuit [6]. Toutes ces données ont été parfaitement résumées en 2008 par Weatherall, qui montrait de plus qu'un processus de sélection naturelle analogue s'était exercé dans pratiquement toutes les hémoglobinopathies majeures, $\mathrm{HbC}, \mathrm{HbE}$, mais aussi dans les $\alpha$-et $\beta$-thalassémies [7], ainsi que dans d'autres pathologies du GR, enzymati-
22. Swartzendruber DC, Wertz PW, Kitko DJ, et al. Molecular models of the intercellular lipid lamellae in mammalian stratum corneum. J Invest Dermatol $1989 ; 92: 251-7$

23. Sandilands $A$, Sutherland $C$, Irvine $A D$, et al. Filaggrin in the frontline: role in skin barrier function and disease. J Cell Sci 2009; 122 : 1285-94.

24. Descargues $P$, Deraison C, Bonnart C, Hovnanian A. Syndrome de Netherton: un modèle d'étude de la régulation de la desquamation. Med Sci (Paris) 2005 $21: 457-8$
Inserm U567-UMR CNRS 8104, Institut Cochin, 24, rue du Faubourg Saint-Jacques,

75014 Paris, France.

dominique.labie@inserm.fr

ques (déficit en glucose-6-phosphate déshydrogénase G6PD) ou membranaires (groupes sanguins, elliptocytose, ovalocytose). Différentes études de populations ont depuis validé cette hypothèse malgré des variations qui seraient dues à une distribution géographique inégale des différents génotypes.

\section{Réinfection \\ de l'anophèle par l'hôte humain}

Sur le plan épidémiologique et clinique le paludisme à $P$. falciparum représente dans les pays d'Afrique subsaharienne un fléau majeur; les accès palustres se comptent par millions, et le nombre de décès annuels, majoritairement des enfants de moins de 5 ans, est de l'ordre du million. Le traitement par la chloroquine, l'usage du DDT, avaient nettement amélioré ces résultats. L'extension progressive de formes du parasite résistantes aux différentes thérapeutiques est un problème croissant d'année en année, l'artémisine et ses dérivés restant encore efficaces sur les formes graves de paludisme $(\rightarrow)$.

La drépanocy-

tose est, dans les

$(\rightarrow)$ Voir la Nouvelle de Vincent Segura, page 701 de ce numéro 


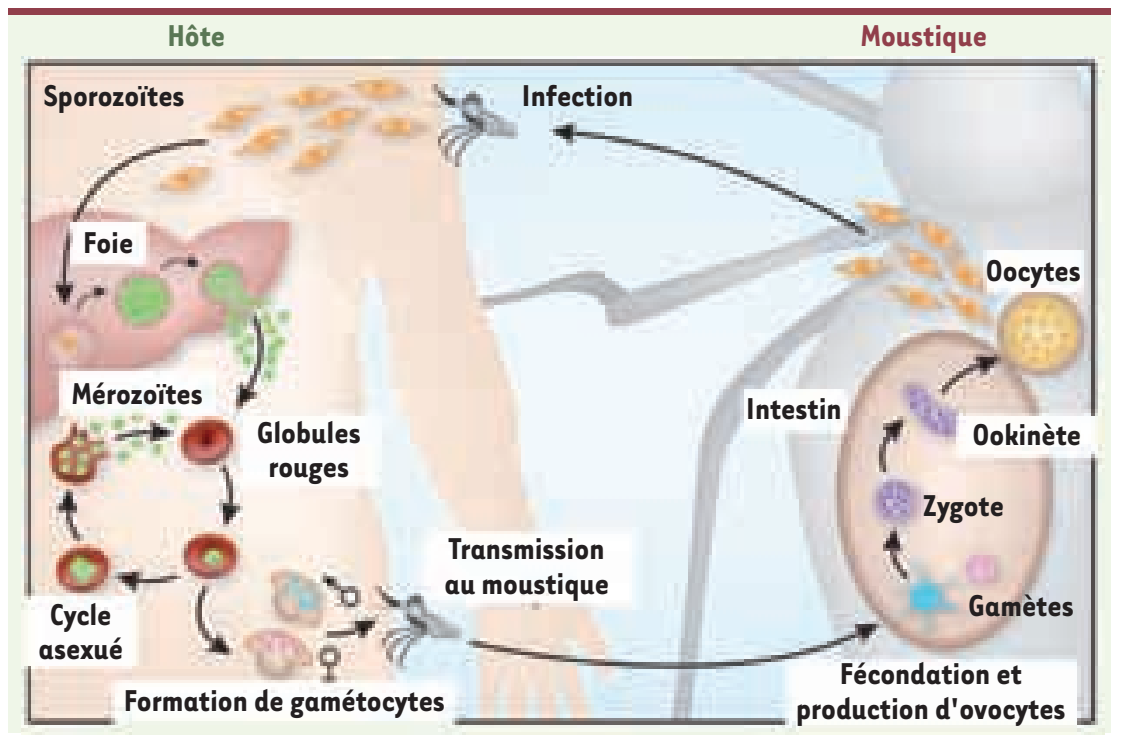

Figure 1. Comment la diversité génétique de l'hôte humain peut influer sur la transmission du Plasmodium au vecteur. L'anophèle femelle transmet les sporozoïtes $P$. falciparum à l'hôte. Après leur maturation dans le foie, les mérozoïtes sont relargués dans la circulation; ils colonisent les globules rouges au cours du cycle asexué ; les gamétocytes mâles et femelles sont produits via la gamétogenèse. Lors de son repas sanguin, un moustique ingère des gamètocytes, qui, dans son intestin, formeront des macro (femelles) ou micro (mâles)gamètes. Après leur fécondation, un ookinète est produit, qui traverse la paroi intestinale et génère des oocystes contenant des sporozoïtes, facilement reconnaissables après la dissection du moustique. Certaines hémoglo-

binopathies sont associées à une protection de l'hôte vis-à-vis de l'infection par $P$. falciparum. Mais ces variants HBB provoquent aussi une transmission accrue des parasites de l'hôte vers le moustique. En présence de mutants $H B B, H b C$ ou $H b S$, le cycle asexué s'épuiserait plus rapidement et la gamétogenèse serait plus précoce. Le prélèvement de ces globules rouges par l'anophèle accélérerait ainsi le développement du cycle sexué chez le vecteur, et la possibilité de réinfection à l'occasion d'une nouvelle piqûre (adapté de [14]).

mêmes pays, la maladie génétique la plus fréquente, posant aussi de difficiles problèmes de prise en charge et responsable de nombreux décès dans l'enfance et à l'adolescence.

Un travail récent effectué au Burkina Faso (ex Haute-Volta) et coordonné par les scientifiques de I'Université La Sapienza à Rome, apporte un élément nouveau d'importance majeure concernant la relation entre la variabilité génétique des $\mathrm{Hb}$ et la transmission entre I'hôte humain et l'anophèle vecteur [8]. Alors que les études antérieures étaient focalisées sur la transmission par l'anophèle du Plasmodium à l'hôte humain, ce dernier travail envisage au contraire le prélèvement par l'anophèle du parasite chez l'homme infecté. Les auteurs rappellent ce qu'on sait du cycle du parasite introduit dans l'organisme par la piqûre de l'anophèle femelle. Après un passage dans le foie, les mérozoïtes libérés dans la circulation s'y reproduisent au cours d'un cycle érythrocytaire asexué qui est seul pathogène [9]. Après un certain nombre de ces cycles, les parasites évoluent vers la formation de gamétocytes mâles et femelles qui seront prélevés par l'anophèle au cours d'un nouveau repas sanguin. Le cycle sexué se déroule alors dans l'organisme du parasite, aboutissant à la formation d'oocytes disponibles pour une nouvelle infection (Figure l).

\section{Infectivité comparée}

\section{de l'hôte selon son génotype HBB}

La présente recherche a exploré l'influence des génotypes de l'hémoglobine (génotype au locus de la $\beta$-globine $H B B$ ) sur cette étape de transmission de I'hôte au vecteur. Les auteurs ont voulu savoir si les variants génétiques, $\mathrm{HbS}$ ou $\mathrm{HbC}$, dont on sait qu'ils sont protecteurs contre les formes graves de paludisme, avaient aussi un impact sur le retour du Plasmodium vers le moustique. L'étude, qui se poursuit depuis 2001 et a inclu 3739 individus, a été épidémiologique, parasitologique et entomologique. Chez I'hôte, on a étudié la fréquence et la densité des formes sexuées de gamétocytes présents dans la circulation et responsables de la transmission à l'anophèle vecteur; ce travail a été effectué in vivo et contrôlé ex vivo. Chez l'anophèle (6 446 insectes) on a exploré le taux et la densité des oocytes après contrôle du génotype sanguin du sujet prélevé par le moustique. Deux enquêtes épidémiologiques ont été menées en milieu rural dans des contextes différents, l'une sur 1737 enfants de moins de 15 ans à la fin de la saison de transmission maximale du paludisme en 2001 , l'autre en 2007 sur 2002 sujets de tous âges à la fin de la saison sèche où la transmission est moindre. La densité de parasites ne variait pas selon le génotype $H B B$ de l'hémoglobine, mais on constatait dans les deux enquêtes un taux de gamétocytes (réservoir de formes sexuées) supérieur dans le sang des sujets porteurs de l'HbC. Une différence (x 4), présente dans les deux séries chez les sujets $C C$, était retrouvée dans la deuxième enquête chez les sujets $A C$; un allèle adaptatif $C$ influerait donc sur la formation des gamétocytes. La différence est nette également si on considère le rapport formes sexuées/asexuées (supérieur de $50 \%$ et $65 \%$ ). Aucune différence significative n'était, en revanche, observée in vivo chez les sujets AS. Bien que l'association entre allèle $C$ et formation de gamétocytes ait été constamment retrouvée, et pour tenir compte des différences d'âge et de populations, les auteurs 
ont effectué une vérification ex vivo. Le degré d'infectivité avait été évalué in vivo en déterminant le taux d'oocytes chez 3869 moustiques disséqués 7 jours après leur repas sanguin chez des hôtes dont on connaissait le génotype $H B B$ : $A A, A S, A C, C C$. La dissection à J3 de $10 \%$ des insectes dans chaque groupe a permis de vérifier un «bruit de fond » identique dans tous les groupes $(P=0,99)$. $\varepsilon$ n revanche, la dissection à $J 7$ identifiait l'origine de la contamination puisqu'elle montrait une densité d'oocytes nettement supérieure quand l'origine de l'infection était un sang contenant l'HbC ( $p=0,002)$; la densité chez les homozygotes était supérieure à celle des hétérozygotes $A C(p=0,004)$. Le risque d'infection de l'anophèle est multiplié par deux s'il se nourrit du sang de sujets porteurs d'HbC, l'infectivité du sang d'individus $A S$, quoique moindre, étant encore supérieure à celle du sang de sujets AA $(p=0,018)$. Le sang des mêmes sujets a été cultivé sur membrane et présenté à des anophèles naïfs, élevés en insectarium, dont ce prélèvement était la première contamination. Le génotype $H B B$ des sangs contrôlés était $A A, A S, A C$ et $C C$. Les résultats constatés chez 2171 moustiques sont encore plus démonstratifs qu'in vivo : le risque d'infection est élevé (x 4) quel que soit le génotype du sang comparé à un sang AA: $\operatorname{AS}(p=0,00015), A C(p=0,0003)$, $C C(p=0,0005)$. On notera que, dans cette procédure in vitro, c'est à partir du sang des sujets AS que le taux d'oocytes mesuré est particulièrement élevé.

\section{Influence du génotype $H B B$}

\section{sur l'efficacité de transmission du} paludisme: hypothèses mécanistiques Cette mise en évidence d'une influence du génotype $H B B$ sur l'efficacité de transmission du paludisme est exemplaire du rôle de la génétique de l'hôte dans la transmission d'une maladie infectieuse. Quels peuvent être les mécanismes cellulaires en cause? Ceux en cause dans la protection de l'hôte ont fait l'objet d'études multiples [7]: ils ont majori- tairement ciblé l'HbS, dont l'impact en santé est majeur en Afrique, mettant en évidence chez les hétérozygotes AS plusieurs mécanismes de protection: diminution de l'invasion parasitaire des $G R$, moindre formation de rosettes et modification de l'adhérence des érythrocytes AS infectés à l'endothélium [10]. Concernant l'HbC, on a montré une moindre expression de l'antigène PfEMP-1 du parasite modifiant l'adhérence à la paroi vasculaire de globules rouges porteurs de cette hémoglobine [11]. Ces mécanismes concernent tous les formes parasitaires asexuées, mais en revanche, on sait peu de choses sur la façon dont le génotype $H B B$ pourrait influencer la formation des gamétocytes en fin de cycle asexué chez l'hôte. La promotion de la différenciation sexuée fait-elle intervenir les mêmes mécanismes que la protection? Des hypothèses ont été faites, plus ou moins anciennes, dont une formation accélérée de gamétocytes en présence de nombres élevés de réticulocytes [12], une modification par les variants $H B B$ de la réponse immunitaire [13], le rôle d'une moindre prise de médicaments par les sujets protégés. On peut envisager une explication plus «finaliste»: il y a un avantage compétitif des formes asexuées sur les formes sexuées, mais en même temps un risque d'extinction. En cas de danger, notamment de perte de l'hôte, nécessaire au cycle du parasite, on peut imaginer un biais vers l'augmentation des formes sexuées, qui serait bénéfique pour le parasite en permettant sa reproduction, et pour l'hôte en diminuant chez lui la densité parasitaire [14].

\section{Conclusion et perspectives}

L'ensemble des résultats du travail de Gougna et al. introduit une nouvelle dimension dans notre approche des interactions entre hémoglobinopathies et gravité du paludisme dans les pays où les deux problèmes sont endémiques. Des variants $H B B$, considérés comme protecteurs, seraient-ils à l'origine d'une aggravation de la transmission du paludisme? La question est d'importance majeure concernant l'HbS étant donné son impact en santé publique. La variabilité des terrains génétiques en Afrique rend nécessaire une étude de terrain similaire dans d'autres pays où l'incidence de l'HbS est un problème dominant, par exemple dans certaines régions de la république démocratique du Congo (RDC) où elle atteint $40 \%$ sans la coexistence d'hémoglobinose $C$. On aimerait aussi savoir si une situation comparable existe dans d'autres pays où existent d'autres variants $H B B$. On pense, entre autres, à l'HbE en Asie du Sud et du Sud-Est. $\diamond$

The complex relations between

haemoglobinopathies and malaria

\section{CONFLIT D'INTÉRÊTS}

L'auteur déclare n'avoir aucun conflit d'intérêts concernant les données publiées dans cet article.

\section{RÉFÉRENCES}

1. Beet $\varepsilon A$. The genetics of the sickle cell trait in a Bantu tribe. Ann Eugenics 1949; 14 : 279-84.

2. Allison AC. Protection afforded by the sickle cell trait against subterian malarial infection. Br Med / 1654 ; $1: 290-4$.

3. Haldane JBS. He rate of mutation of human genes. Proceedings of the VIII ${ }^{\text {th }}$ International Congress of Genetics. Hereditas $1949 ; 35: 267-73$.

4. Luzzatto L, Nwachiku-Jarrett $\& S$, Reddy S. Increased sickling of parasited erythrocytes as mechanism of resistance against malaria in sickle-cell trait. Lancet $1970 ; 1$ : 319

5. Roth દદ, Friedman M, Ueda Y, et al. Sickling 1 rates of human AS red cells infected in vitro with Plasmodium falciparum malaria. Science $1978: 202: 650-2$.

6. Friedman ML, Roth $\varepsilon \varepsilon$, Nagel RL, Trager W. Plasmodium falciparum physiological interctions with the human sickle cell. Exp Parasitol $1979 ; 4: 73-80$.

7. Weatherall DJ. Genetic variation ans susceptibiility to infection : the red cell and malaria. BrJ Haematol 2008 $141: 276-86$

8. Gouagna LC, Bancone G, Yao F, et al. Genetic variation in human HBB is associated with Plasmodium falciparum transmission. Nat Genet $2010 ; 42$ : 328-31.

9. Pasvol G, Weatherall DJ. Cellular mechanism for the protective effect of haemoglobin $S$ against $P$. falciparum malaria. Nature $1978 ; 274: 701-3$.

10. Cholera R, Brittain NJ, Gillrie MR, et al. Impaired cytoadherence of Plasmodium falciparum-infected erythrocytes containing sickle hemoglobin. Proc Natl Acad Sci USA 2008 ; 105 : 991-6.

11. Fairhurst RM, Baruch DL, Brittain NJ, et al. Abnormal display of PfEMP-1 on erythrocytes carrying haemoglobin $C$ may protect againt malaria. Nature 2005 ; 435 : 1117-21.

12. Trager W, Gill GS, Lawrence C, Nagel RL. Plasmodium falciparum enhanced gamétocyte formation in vitro in reticulocyte-rich blood. Exp Parasitol $1999 ; 91$ : 115-8.

13. Verrra F, Simpura J, Warimwe GM, et al. Haemoglobin C and $S$ role in acquired immunity against Plasmodium falciparum malaria. PloS One 2007 ; 2 : e978.

14. Pasvol G. Protective haemoglobinopathies and Plasmodium falciparum transmission. Nat Genet $2010 ; 42: 284-5$. 